\title{
Cortical construction: from molecules to models
}

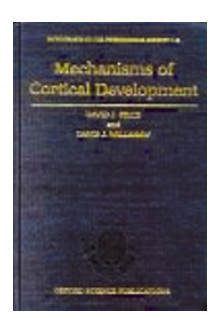

\author{
Mechanisms of Cortical Development
}

by David J. Price and David J. Willshaw

Oxford University Press, New York, 2000. \$59.95

hardcover, pp 315

ISBN 0-19-262427-X

\section{Reviewed by Linda J. Richards and Geoffrey J. Goodhill}

The cortex underlies the most sophisticated feats of animals' sensory processing and behavior. Humans have the largest ratio of cortical area to body size of any animal, and the cortex controls uniquely human behaviors such as language. How a structure of such computational power is constructed during development has fascinated both biologists interested in development, and theoreticians interested in biologically inspired computing machines.

The cortex's superficially uniform anatomical structure and repetitive organization into columns has suggested that just a few general rules can explain its development. An attractive hypothesis (especially to theoreticians in search of parsimony, elegance and ways to apply artificial neural network models) is that although early stages of cortical development are governed by molecular mechanisms relatively immune to neural activity, later stages are largely driven by afferent input. So, for instance, visual cortex becomes visual in structure and function mostly because it receives visual inputs rather than, say, auditory inputs. Theoretical work has generally focused on later stages, and in areas such as map development in visual cortex, theoretical and experimental researchers have established an active dialogue.

However, much has changed in our understanding of neural development over the past decade or so, and modern molecular techniques have enabled the study of activity-independent mechanisms of

Linda Richards is at the University of Maryland, Baltimore, Department of Anatomy and Neurobiology and the Program in Neuroscience, 685 West Baltimore Street, Baltimore,

Maryland 21201, USA.

Geoffrey Goodhill is in the Department of Neuroscience, Georgetown University Medical Center, 3900 Reservoir Road, Washington, D.C. 20007, USA. development at an unprecedented level of detail. It is now apparent that molecules expressed early in development exert strong control over how the cortex initially becomes functionally regionalized. For instance, in a mutant mouse lacking the transcription factor Gbx-2, thalamic axons stall in the internal capsule and do not reach the cortex, yet regionalization of the neocortex still occurs, at least at the level of region-specific gene expression. Beyond simple reaction-diffusion schemes, there are virtually no mathematical theories that address how networks of gene expression control early cortical regionalization.

Mechanisms of Cortical Development tries to combine experimental and theoretical perspectives of cortical development. It reflects a growing trend toward bridging the gap between the two approaches of study: describing structure and its formation and maintenance, and analyzing function and its relationship to structure. The authors, one an experimentalist and one a theoretician, are longtime colleagues at the University of Edinburgh.

The book follows development of the cortex from the earliest events in the molecular regulation of neural induction to models of map formation, receptive field properties and, very briefly, higher cognitive functions. Much of the book is necessarily molecular and empirical, given the recent expansion of knowledge in this area. However, the focus is more on concepts rather than on molecules, although key molecules are listed in tables and diagrams. In Chapter 2, the authors give details of cell differentiation along with definitions of concepts such as cellular specification, determination and potency. Molecular regulation of regional specification of the cortex is described in Chapter 3 within the context of cellular processes such as proliferation, migration and differentiation. Mechanisms of axon guidance are discussed in Chapter 4; key concepts of attraction, repulsion and growth cone dynamics are explained within a molecular framework. These chapters are up-to-date and relevant, and provide both a general overview and the bibliographical resources to explore these concepts in more detail.

Throughout, descriptions of theoretical models are written in an intuitive style mostly free of equations. To accommodate a wide audience, the authors detail some concepts relevant to developmental neuroscience, such as mutant mouse technologies, and the life cycle of retroviruses. However, other techniques well-suited for studying cortical development are not described, such as optical techniques for imaging maps and intrinsic circuits, and 'second-generation' knockout technologies utilizing Cre/loxP or Flp/frtd recombination. Each chapter incorporates subtitles, making it easy to find a specific topic, molecule or structure. The book would therefore be appropriate as a prescribed text for graduate courses, although all the figures are grayscale, making the book less attractive for teaching purposes than some other developmental neuroscience texts.

A challenge facing anyone trying to review cortical development is that relatively little is known about this topic compared to development elsewhere in the nervous system. The authors reasonably assume that similarities may exist, and that an appreciation of mechanisms of noncortical development (both within the nervous system and elsewhere) can be important for understanding cortical development. For example, map formation in the retinotectal system and synapse elimination (although not synapse formation) at the neuromuscular junction are covered. However, the relevance of these data to cortical development is left to the reader to judge.

Data about cortical development are accumulating rapidly, and several advances have been made since this book went to press. The speed at which we can now address these issues prevents any text from remaining current for long. Theoretical models are particularly vulnerable to being disproved in next month's Nature Neuroscience. However, even if proven incorrect for a particular problem, good models remain powerful tools that can be relevant to other situations (for example, reaction-diffusion models). This book represents a step toward a closer dialogue between theoreticians and experimentalists, a step that can only further our understanding of the brain. 